\title{
LOCALIZATION FOR RIESZ MEANS OF FOURIER EXPANSIONS
}

\author{
LEONARDO COLZANI, GIACOMO GIGANTE, AND ANA VARGAS
}

\begin{abstract}
The classical Riemann localization principle states that if an integrable function of one variable vanishes in an open set, then its Fourier expansion converges to zero in this set. This principle does not immediately extend to several dimensions, and here we study the Hausdorff dimension of the sets of points where localization for Riesz means of Fourier expansions may fail.
\end{abstract}

The Fourier transform and Fourier inversion formula are defined by

$$
\widehat{f}(\xi)=\int_{\mathbb{R}^{d}} f(x) \exp (-2 \pi i \xi x) d x, \quad f(x)=\int_{\mathbb{R}^{d}} \widehat{f}(\xi) \exp (2 \pi i \xi x) d \xi .
$$

Since these integrals may not be absolutely convergent, a number of summation methods have been introduced; one of these is the Riesz means:

$$
\begin{gathered}
S_{R}^{\alpha} * f(x)=\int_{\{|\xi| \leq R\}}\left(1-|\xi|^{2} / R^{2}\right)^{\alpha} \widehat{f}(\xi) \exp (2 \pi i \xi x) d \xi \\
=\int_{\mathbb{R}^{d}} \pi^{-\alpha} \Gamma(\alpha+1) R^{d / 2-\alpha}|y|^{-\alpha-d / 2} J_{\alpha+d / 2}(2 \pi R|y|) f(x-y) d y .
\end{gathered}
$$

The multiplier $\left(1-|\xi|^{2} / R^{2}\right)_{+}^{\alpha}$ is a damping for high frequencies, and the higher the exponent $\alpha$, the smoother the multiplier, and the more efficient the summation method. In particular, when $\alpha=0$, one obtains the so-called spherical partial sums. See [24, Chapter IV]. The classical Riemann localization principle states that if an integrable function of one variable vanishes in an open set, then the partial sums of its Fourier expansion converge uniformly to zero in every compact subset of the open set. It has been observed by Tonelli that this localization principle does not immediately extend from dimension one to dimension $d>1$, and Bochner has proved that localization may fail for Riesz means with $\alpha<(d-1) / 2$, while it holds above this critical index; that is, if $\alpha \geq(d-1) / 2$, then $\lim _{R \rightarrow+\infty}\left\{S_{R}^{\alpha} * f(x)\right\}=0$ at all points in an open set where the function vanishes. Indeed, these results are corollaries of the Banach-Steinhaus theorem. If $1 \leq p, q \leq+\infty$ and $1 / p+1 / q=1$, the norms of the linear functionals $S_{R}^{\alpha} * f(x)$ on $p$ integrable functions vanishing in $\{|y-x| \leq \varepsilon\}$ are the $q$ norms of the kernels $S_{R}^{\alpha}(y)$ in $\{|y|>\varepsilon\}$. Since these kernels have sizes $R^{(d-1) / 2-\alpha}|y|^{-(d+1) / 2-\alpha}$, the functionals are uniformly bounded if and only if $\alpha \geq(d-1) / 2$. Indeed, in [14] and [20, it is shown through explicit

Received by the editors August 31, 2012.

2010 Mathematics Subject Classification. Primary 42B08; Secondary 28A78.

Key words and phrases. Sets of divergence, localization, Riesz means, Hausdorff dimension.

The third author was partially supported by Grant MTM2010-16518, Ministerio de Economía y Competitividad, Spain. 
examples that pointwise localization may fail even for quite nice functions: If $d \geq 3$, then $S_{R}^{0} * \chi_{\{|x|<1\}}(0)$ does not converge. Despite these negative results, it has been proved in [3] and [6] that for spherical partial sums of square integrable functions localization holds almost everywhere; that is, at almost all points of an open set where a square integrable function vanishes one has $\lim _{R \rightarrow+\infty}\left\{S_{R}^{0} * f(x)\right\}=0$. See also [2, 17, 18] for previous analogues for spherical harmonic expansions, and [10,11 for alternative proofs. It is known that nice functions have better properties than almost everywhere convergence. In particular, Beurling and then Salem and Zygmund (see [25, Chapter XIII]), studied the capacity of sets of divergence of onedimensional Fourier series of functions in Sobolev classes, and these results have been extended to multidimensional Fourier expansions in [7,8, 12, 19. In particular, in [19] it is proved that the spherical partial sums $S_{R}^{0} * f(x)$ of functions in a Sobolev space $L^{\gamma, p}\left(\mathbb{R}^{d}\right)$, with $0<\gamma \leq d / p$ and $2 \leq p<2 d /(d-1)$, converge $C_{\gamma-\varepsilon, 2}$ quasieverywhere for every $0<\varepsilon<\gamma$, and localization holds also with $\varepsilon=0$.

Continuing this line of research in the area of exceptional sets in harmonic analysis, here we study the convergence of Riesz means of functions which are locally smooth, but not globally in Sobolev classes. Our purpose is to fill the gap and interpolate between the pointwise result at $\alpha=(d-1) / 2$ and the almost everywhere result at $\alpha=0$. In particular, we would like to prove that when $0<\alpha<(d-1) / 2$, then localization holds with a possible exception in a set of points of dimension $0<\delta<d$. As usual, instead of the $\operatorname{limit}_{R \rightarrow+\infty}\left\{S_{R}^{\alpha} * f(x)\right\}$, it is convenient to consider the maximal operator $\sup _{R>0}\left\{\left|S_{R}^{\alpha} * f(x)\right|\right\}$. More generally, one can consider $\sup _{R>0}\left\{R^{-\beta}\left|S_{R}^{\alpha} * f(x)\right|\right\}$, for some $-\infty<\beta<+\infty$. This parameter, when positive, measures the speed of divergence and, when negative, the speed of convergence. In what follows $f(x)$ is a tempered distribution in the Sobolev space $H^{\gamma}\left(\mathbb{R}^{d}\right)$, with $-\infty<\gamma<+\infty$, defined by the norm

$$
\left\{\int_{\mathbb{R}^{d}}\left(1+|\xi|^{2}\right)^{\gamma}|\widehat{f}(\xi)|^{2} d x\right\}^{1 / 2}<+\infty .
$$

Indeed, it turns out that all these indices $\alpha, \beta, \gamma$ are somehow redundant, and what really counts is the quantity $\alpha+\beta+\gamma$.

Theorem 1. Fix an $\varepsilon>0$, and assume that one of the following holds:

(1) $\alpha \geq 0, \alpha+\beta+\gamma \geq(d-1) / 4, \delta \geq d-2(\alpha+\beta+\gamma)-1 \geq 0$,

(2) $\alpha>0, \alpha+\beta+\gamma>0, \delta>d-2(\alpha+\beta+\gamma) \geq 0$.

Then there exists a positive constant $c$ with the property that for every tempered distribution $f(x)$ vanishing in an open set $\Omega$ and for every nonnegative Borel measure $d \mu(x)$ with support in $\Omega \cap\{$ distance $\{x, \partial \Omega\}>\varepsilon\}$,

$$
\begin{gathered}
\int_{\mathbb{R}^{d}} \sup _{R>0}\left\{R^{-\beta}\left|S_{R}^{\alpha} * f(x)\right|\right\} d \mu(x) \\
\leq c\left\{\int_{\mathbb{R}^{d}}\left(1+|\xi|^{2}\right)^{\gamma}|\widehat{f}(\xi)|^{2} d \xi\right\}^{1 / 2}\left\{\int_{\mathbb{R}^{d}} \int_{\mathbb{R}^{d}} \frac{d \mu(x) d \mu(y)}{|x-y|^{\delta}}\right\}^{1 / 2} .
\end{gathered}
$$

(3) Finally, if $\alpha \geq 0$ and $\alpha+\beta+\gamma \geq(d-1) / 2$, then

$\sup _{R>0, x \in \Omega \cap\{\text { distance }\{x, \partial \Omega\}>\varepsilon\}}\left\{R^{-\beta}\left|S_{R}^{\alpha} * f(x)\right|\right\} \leq c\left\{\int_{\mathbb{R}^{d}}\left(1+|\xi|^{2}\right)^{\gamma}|\widehat{f}(\xi)|^{2} d \xi\right\}^{1 / 2}$. 
By the above theorem, the means $R^{-\beta}\left|S_{R}^{\alpha} * f(x)\right|$ cannot diverge on the supports of measures with finite energy. Hence, by the relations between energy, capacity, and dimension, these means cannot diverge on sets with large dimension.

Corollary 2. Assume that a tempered distribution $f(x)$ in the Sobolev space $H^{\gamma}\left(\mathbb{R}^{d}\right)$ vanishes in an open set $\Omega$, and also assume one of the following:

(1) $\alpha \geq 0, \alpha+\beta+\gamma \geq(d-1) / 4, \delta=d-2(\alpha+\beta+\gamma)-1$,

(2) $\alpha>0, \alpha+\beta+\gamma>0, \delta=d-2(\alpha+\beta+\gamma)$.

Then $\lim _{R \rightarrow+\infty}\left\{R^{-\beta} S_{R}^{\alpha} * f(x)\right\}=0$ at all points in the open set $\Omega$, with possible exceptions in a set with Hausdorff dimension at most $\delta$.

(3) Finally, if $\alpha \geq 0$ and $\alpha+\beta+\gamma \geq(d-1) / 2$, then $\lim _{R \rightarrow+\infty}\left\{R^{-\beta} S_{R}^{\alpha} * f(x)\right\}$ $=0$ at all points in $\Omega$.

In the above theorem and corollary the estimate (1) $\delta \geq d-2(\alpha+\beta+\gamma)-1$ is better than (2) $\delta>d-2(\alpha+\beta+\gamma)$, but is limited to the interval $\alpha+\beta+\gamma \geq$ $(d-1) / 4$. We do not know if these indices are best possible, however, observe that the natural conjecture given by the linear interpolation between the almost everywhere result $(\alpha+\beta+\gamma, \delta)=(0, d)$ and the pointwise result $(\alpha+\beta+\gamma, \delta)=$ $((d-1) / 2,0)$, at least when $\alpha+\beta+\gamma \geq(d-1) / 4$ is worse than the above results.

By the Sobolev embedding, if $1 \leq p<2$ and $\gamma<d(1 / 2-1 / p)$, or $1<p<2$ and $\gamma \leq d(1 / 2-1 / p)$, then

$$
\left\{\int_{\mathbb{R}^{d}}\left(1+|\xi|^{2}\right)^{\gamma}|\widehat{f}(\xi)|^{2} d x\right\}^{1 / 2} \leq c\left\{\int_{\mathbb{R}^{d}}|f(x)|^{p} d x\right\}^{1 / p} .
$$

See [22, Chapter V]. Hence the above theorem and corollary immediately give a localization result for the Lebesgue spaces $L^{p}\left(\mathbb{R}^{d}\right)$ with $p \leq 2$. It is stated in [7.9] that at the indices $\alpha=\beta=\gamma=0$ localization may fail on sets of measure zero but of full dimension. For related results see also 1, 1, 12,19. The next result generalizes these counterexamples, with $\gamma=0$, which is the case of square integrable functions.

Theorem 3. Assume that $d \geq 2$ and $1<\delta \leq d-4(\alpha+\beta)$, and assume that the set $E$ is contained in the intersection of the ball $\{|x|<1\}$ with the product domain $(-1,1) \times E^{\prime}$, with $E^{\prime} \subset(-1,1)^{d-1}$ and $E^{\prime}$ of $\delta-1$ dimensional measure 0 . Then there is a square integrable function $f(x)$ which vanishes in $\{|x|<2\}$ and such that $\sup _{R>0}\left\{R^{-\beta}\left|S_{R}^{\alpha} * f(x)\right|\right\}=+\infty$ for every $x$ in $E$.

In particular, there exist sets with dimension $d-4 \alpha$ which are of nonlocalization for some square integrable functions, and every set with $d-4 \alpha-1$-dimensional measure zero is of nonlocalization. As said before, we do not know if these results are sharp, however, observe that Theorem 1 with $d=2$ and $\beta=\gamma=0$ and $\alpha=1 / 4$ gives localization up to sets of dimension $1 / 2$, while Theorem 3 with $d=2$ and $\beta=\gamma=0$ and $\alpha<1 / 4$ gives nonlocalization in sets of dimension $2-4 \alpha>1$. Hence at $\alpha=(d-1) / 4$ with $\beta=\gamma=0$ and $d=2$ there is a real discontinuity.

Proof of Theorem 1. The main tool in what follows is a decomposition of the Riesz means into a convolution with a kernel with compact support and a kernel with small Fourier transform. Let $S_{R}^{\alpha}(x)$ be the kernel of the Riesz means,

$$
\begin{gathered}
\widehat{S_{R}^{\alpha}}(\xi)=\left(1-|\xi|^{2} / R^{2}\right)_{+}^{\alpha}, \\
S_{R}^{\alpha}(x)=\pi^{-\alpha} \Gamma(\alpha+1) R^{d / 2-\alpha}|y|^{-\alpha-d / 2} J_{\alpha+d / 2}(2 \pi R|y|) .
\end{gathered}
$$




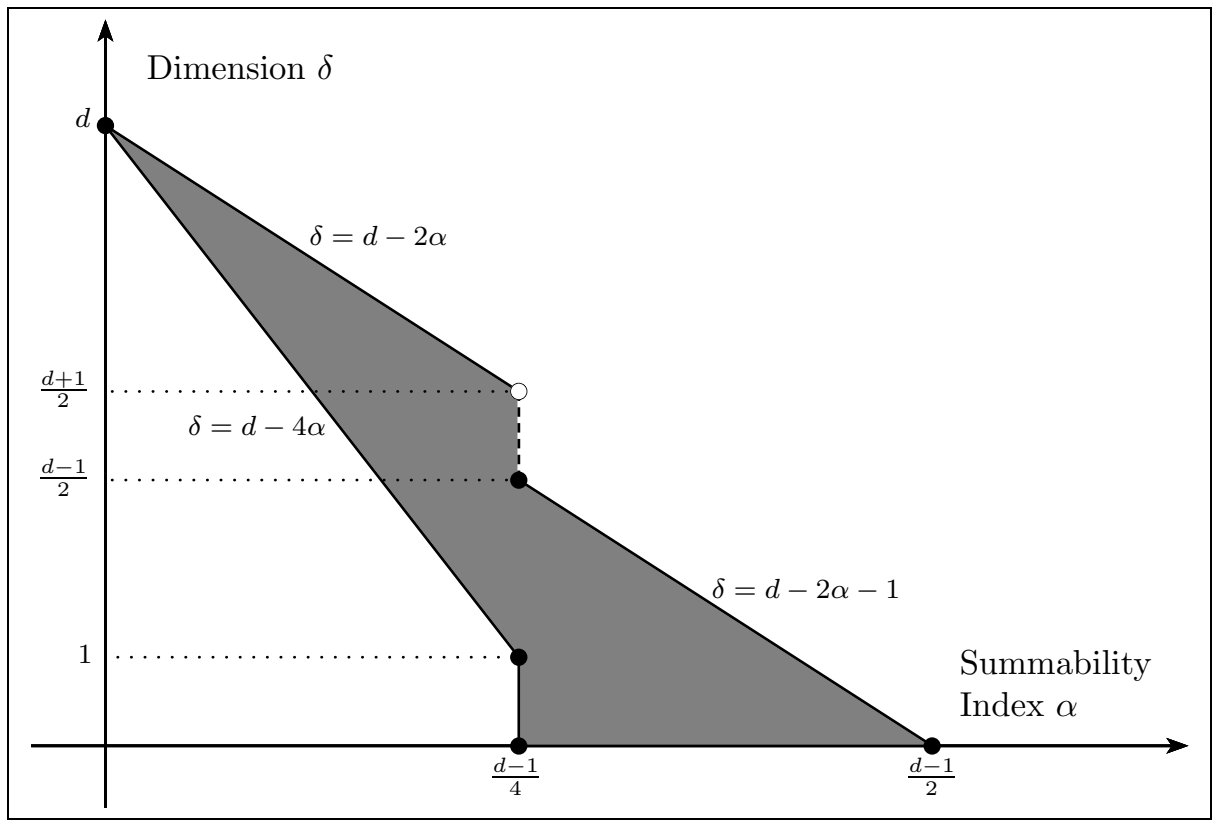

Figure 1. The upper (Theorem 1) and lower (Theorem 3) bounds for the maximal dimension $\delta$ of a set of nonlocalization for Riesz means of order $\alpha$ of a function in $L^{2}\left(\mathbb{R}^{d}\right)$.

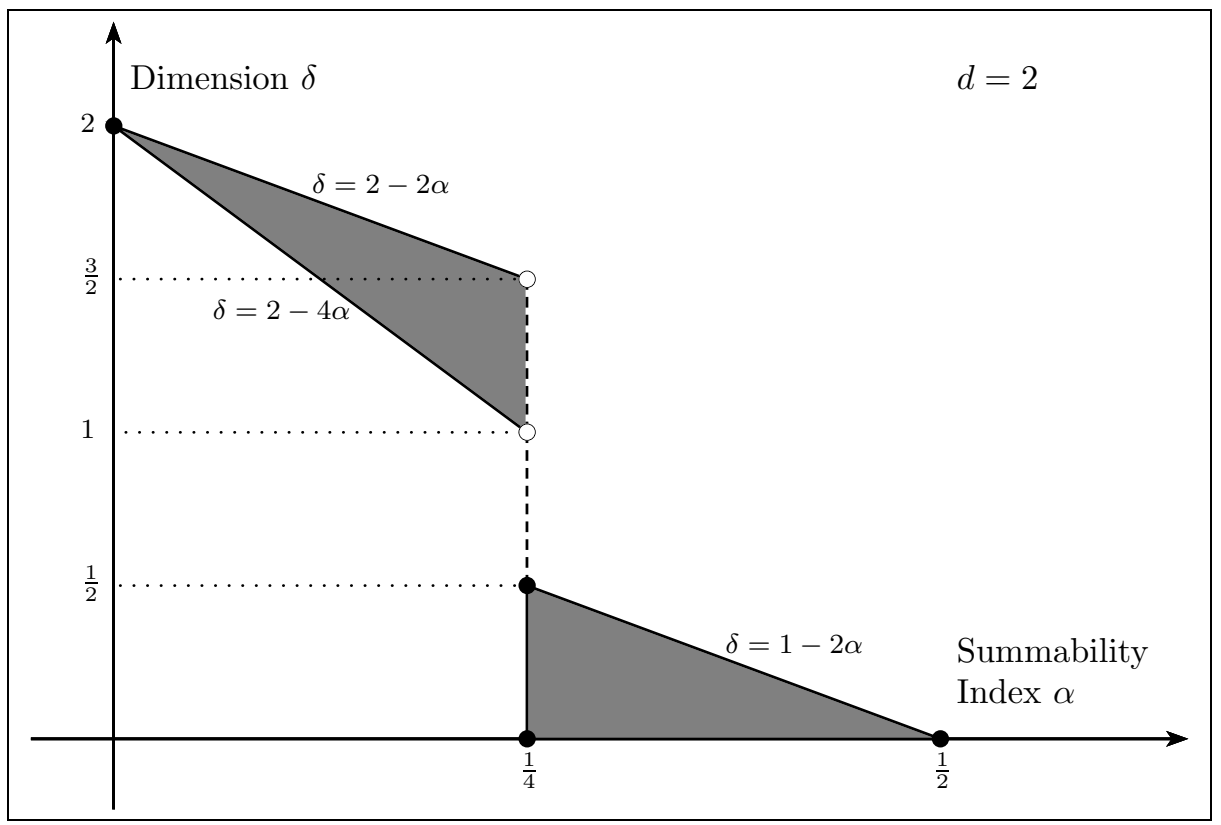

FiguRE 2. The 2-dimensional case. 
Also, let $\varepsilon>0$ and let $\psi(x)$ be a radial nonnegative smooth function with $\psi(x)=1$ if $|x| \leq \varepsilon / 2$ and $\psi(x)=0$ if $|x| \geq \varepsilon$. Then

$$
R^{-\beta} S_{R}^{\alpha}(x)=R^{-\beta} \psi(x) S_{R}^{\alpha}(x)+R^{-\beta}(1-\psi(x)) S_{R}^{\alpha}(x)=A_{R}(x)+B_{R}(x) .
$$

In order to prove the theorem it suffices to show that $A_{R} * f(x)=0$ for every $x$ in $\Omega$ with distance $(x, \partial \Omega)>\varepsilon$, and that the maximal function $\sup _{R>1}\left\{\left|B_{R} * f(x)\right|\right\}$ can be integrated against a measure $d \mu(x)$ with finite energy.

Lemma 4. If $f(x)$ vanishes in an open set $\Omega$, then for all $x$ in $\Omega$ with distance $\{x, \partial \Omega\}>\varepsilon$,

$$
A_{R} * f(x)=0
$$

Proof. If $A_{R}(x)$ has support in $\{|x| \leq \varepsilon\}$, and if $f(x)$ has support in a set $\mathbb{R}^{d}-\Omega$, then $A_{R} * f(x)$ has support in $\left\{\right.$ distance $\left.\left\{x, \mathbb{R}^{d}-\Omega\right\} \leq \varepsilon\right\}$, and this set does not intersect $\Omega \cap\{$ distance $\{x, \partial \Omega\}>\varepsilon\}$.

Lemma 5. For every $k>0$, there exists $c>0$ such that for every $\xi$ in $\mathbb{R}^{d}$ and every $R>1$,

$$
\left|\widehat{B_{R}}(\xi)\right| \leq c R^{-\alpha-\beta}(1+|R-| \xi||)^{-k}
$$

Proof. The idea is that $\widehat{A_{R}}(\xi)=R^{-\beta} \widehat{\psi} * \widehat{S_{R}^{\alpha}}(\xi)$ is an approximation of $R^{-\beta} \widehat{S_{R}^{\alpha}}(\xi)$, and away from the singularities of this function in $|\xi|=R$ the approximation is particularly good. First observe that $R-|\xi-\zeta| \leq|R-| \xi||+|\zeta|$, and that

$$
\widehat{S_{R}^{\alpha}}(\xi-\zeta)=R^{-2 \alpha}(R+|\xi-\zeta|)^{\alpha}(R-|\xi-\zeta|)_{+}^{\alpha} \leq 2^{\alpha} R^{-\alpha}(|R-| \xi||+|\zeta|)^{\alpha} .
$$

Also observe that for every multi-index $n$ there exists a polynomial $P_{n}(\xi)$ of degree $|n|$ such that

$$
\frac{\partial^{n}}{\partial \xi^{n}}\left(1-\left|R^{-1} \xi\right|^{2}\right)_{+}^{\alpha}=R^{-|n|} P_{n}\left(R^{-1} \xi\right)\left(1-\left|R^{-1} \xi\right|^{2}\right)_{+}^{\alpha-|n|}
$$

Hence, for some constant which may depend on the multi-index,

$$
\left|\frac{\partial^{n}}{\partial \xi^{n}}\left(1-|\xi|^{2} / R^{2}\right)_{+}^{\alpha}\right| \leq\left. C R^{-\alpha}|R-| \xi\right|^{\alpha-|n|} .
$$

Since $\widehat{\psi}(\xi)$ has rapid decay at infinity, if $|R-| \xi|| \leq 1$, then

$$
\begin{gathered}
R^{\beta}\left|\widehat{B_{R}}(\xi)\right|=\left|\widehat{S_{R}^{\alpha}}(\xi)-\widehat{\psi} * \widehat{S_{R}^{\alpha}}(\xi)\right| \leq\left|\widehat{S_{R}^{\alpha}}(\xi)\right|+\left|\widehat{\psi} * \widehat{S_{R}^{\alpha}}(\xi)\right| \\
\leq\left. 2^{\alpha} R^{-\alpha}|R-| \xi\right|^{\alpha}+2^{\alpha} R^{-\alpha} \int_{\mathbb{R}^{d}}(|R-\xi|+|\zeta|)^{\alpha}|\widehat{\psi}(\zeta)| d \zeta \leq C R^{-\alpha} .
\end{gathered}
$$


Now consider the case $|R-| \xi|| \geq 1$. Since $\widehat{\psi}(\xi)$ has mean one and all other moments are zero,

$$
\begin{gathered}
R^{\beta}\left|\widehat{B_{R}}(\xi)\right|=\left|\widehat{S_{R}^{\alpha}}(\xi)-\widehat{\psi} * \widehat{S_{R}^{\alpha}}(\xi)\right| \\
=\left|\int_{\mathbb{R}^{d}}\left(\left(1-|\xi-\zeta|^{2} / R^{2}\right)_{+}^{\alpha}-\sum_{|n|<N} \frac{\partial^{n}}{\partial \xi^{n}}\left(1-|\xi|^{2} / R^{2}\right)_{+}^{\alpha} \frac{(-\zeta)^{n}}{n !}\right) \widehat{\psi}(\zeta) d \zeta\right| \\
\leq \int_{\{|\zeta| \geq|R-| \xi|| / 2\}}\left(1-|\xi-\zeta|^{2} / R^{2}\right)_{+}^{\alpha}|\widehat{\psi}(\zeta)| d \zeta \\
+\sum_{|n|<N} \frac{1}{n !}\left|\frac{\partial^{n}}{\partial \xi^{n}}\left(1-|\xi|^{2} / R^{2}\right)_{+}^{\alpha}\right| \int_{\{|\zeta| \geq|R-| \xi|| / 2\}}\left|\zeta^{n}\right||\widehat{\psi}(\zeta)| d \zeta \\
+\sum_{|n|=N} \frac{1}{n !} \sup _{\{|\zeta| \leq|R-| \xi|| / 2\}}\left\{\left|\frac{\partial^{n}}{\partial \xi^{n}}\left(1-|\xi-\zeta|^{2} / R^{2}\right)_{+}^{\alpha}\right|\right\} \int_{\{|\zeta| \leq|R-| \xi|| / 2\}}\left|\zeta^{n}\right||\widehat{\psi}(\zeta)| d \zeta .
\end{gathered}
$$

The integrals over $\{|\zeta| \geq|R-| \xi|| / 2\}$ can be estimated as follows:

$$
\begin{gathered}
\int_{\{|\zeta| \geq|R-| \xi|| / 2\}}\left(1-|\xi-\zeta|^{2} / R^{2}\right)_{+}^{\alpha}|\widehat{\psi}(\zeta)| d \zeta \\
\leq 2^{\alpha} R^{-\alpha} \int_{\{|\zeta| \geq|R-| \xi|| / 2\}}(|R-\xi|+|\zeta|)^{\alpha}|\widehat{\psi}(\zeta)| d \zeta \\
\leq 6^{\alpha} R^{-\alpha} \int_{\{|\zeta| \geq|R-| \xi|| / 2\}}|\zeta|^{\alpha}|\widehat{\psi}(\zeta)| d \zeta \leq C R^{-\alpha}|R-\xi|^{-k} \\
\left|\frac{\partial^{n}}{\partial \xi^{n}}\left(1-|\xi|^{2} / R^{2}\right)_{+}^{\alpha}\right| \int_{\{|\zeta| \geq|R-| \xi|| / 2\}}\left|\zeta^{n}\right||\widehat{\psi}(\zeta)| d \zeta \\
\leq C R^{-\alpha}|R-| \xi||^{\alpha-|n|} \int_{\{|\zeta| \geq|R-| \xi|| / 2\}}\left|\zeta^{n}\right||\widehat{\psi}(\zeta)| d \zeta \leq C R^{-\alpha}|R-\xi|^{-k}
\end{gathered}
$$

The integrals over $\{|\zeta| \leq|R-| \xi|| / 2\}$ and with $|n|=N$ can be estimated as follows:

$$
\begin{gathered}
\sup _{\{|\zeta| \leq|R-| \xi|| / 2\}}\left\{\left|\frac{\partial^{n}}{\partial \xi^{n}}\left(1-|\xi-\zeta|^{2} / R^{2}\right)_{+}^{\alpha}\right|\right\} \int_{\{|\zeta| \leq|R-| \xi|| / 2\}}\left|\zeta^{n}\right||\widehat{\psi}(\zeta)| d \zeta \\
\leq\left. C R^{-\alpha}|R-| \xi\right|^{\alpha-N} \int_{\mathbb{R}^{d}}|\zeta|^{N}|\widehat{\psi}(\zeta)| d \zeta \leq C R^{-\alpha}|R-\xi|^{\alpha-N} .
\end{gathered}
$$

The desired result follows by taking $N \geq \alpha+k$.

As usual, it is convenient to replace the maximal operator $\sup _{R>1}\left\{\left|B_{R}^{\alpha} * f(x)\right|\right\}$ with a linearized version $B_{R(x)} * f(x)$, with $R(x)$ an arbitrary Borel function. In what follows, the support of the distribution $f(x)$ will play no role.

Lemma 6. If $(d-1) / 4 \leq \alpha+\beta+\gamma \leq(d-1) / 2$, then there exists a constant $c>0$ with the property that for every distribution $f(x)$ in $H^{\gamma}\left(\mathbb{R}^{d}\right)$, for every Borel 
function $R(x)>1$, and for every nonnegative finite Borel measure $d \mu(x)$,

$$
\begin{gathered}
\int_{\mathbb{R}^{d}}\left|B_{R(x)} * f(x)\right| d \mu(x) \\
\leq c\left\{\int_{\mathbb{R}^{d}}\left(1+|\xi|^{2}\right)^{\gamma}|\widehat{f}(\xi)|^{2} d x\right\}^{1 / 2}\left\{\int_{\mathbb{R}^{d}} \int_{\mathbb{R}^{d}} \frac{d \mu(x) d \mu(y)}{|x-y|^{d-2(\alpha+\beta+\gamma)-1}}\right\}^{1 / 2} .
\end{gathered}
$$

Similarly, if $\alpha+\beta+\gamma \geq(d-1) / 2$, then

$$
\begin{gathered}
\int_{\mathbb{R}^{d}}\left|B_{R(x)} * f(x)\right| d \mu(x) \\
\leq c\left\{\int_{\mathbb{R}^{d}}\left(1+|\xi|^{2}\right)^{\gamma}|\widehat{f}(\xi)|^{2} d x\right\}^{1 / 2}\left\{\int_{\mathbb{R}^{d}} \int_{\mathbb{R}^{d}} d \mu(x) d \mu(y)\right\}^{1 / 2} .
\end{gathered}
$$

Proof. If $g(x)=\overline{B_{R(x)} * f(x)} /\left|B_{R(x)} * f(x)\right|$, then

$$
\begin{gathered}
\int_{\mathbb{R}^{d}}\left|B_{R(x)} * f(x)\right| d \mu(x) \\
=\int_{\mathbb{R}^{d}} \int_{\mathbb{R}^{d}} g(x) \widehat{B_{R(x)}}(\xi) \widehat{f}(\xi) \exp (2 \pi i \xi x) d \xi d \mu(x) \\
\leq\left\{\int_{\mathbb{R}^{d}}\left(1+|\xi|^{2}\right)^{\gamma}|\widehat{f}(\xi)|^{2} d \xi\right\}^{1 / 2} \\
\times\left\{\int_{\mathbb{R}^{d}}\left(1+|\xi|^{2}\right)^{-\gamma}\left|\int_{\mathbb{R}^{d}} g(x) \widehat{B_{R(x)}}(\xi) \exp (2 \pi i \xi x) d \mu(x)\right|^{2} d \xi\right\}^{1 / 2} .
\end{gathered}
$$

Moreover,

$$
\begin{aligned}
& \int_{\mathbb{R}^{d}}\left(1+|\xi|^{2}\right)^{-\gamma}\left|\int_{\mathbb{R}^{d}} g(x) \widehat{B_{R(x)}}(\xi) \exp (2 \pi i \xi x) d \mu(x)\right|^{2} d \xi \\
& =\int_{\mathbb{R}^{d}} \int_{\mathbb{R}^{d}} \int_{\mathbb{R}^{d}}\left(1+|\xi|^{2}\right)^{-\gamma} \widehat{B_{R(x)}}(\xi) \widehat{B_{R(y)}}(\xi) \exp (2 \pi i(x-y) \xi) g(x) \overline{g(y)} d \mu(x) d \mu(y) d \xi \\
& \leq \int_{\mathbb{R}^{d}} \int_{\mathbb{R}^{d}}\left|\int_{\mathbb{R}^{d}}\left(1+|\xi|^{2}\right)^{-\gamma} \widehat{B_{R(x)}}(\xi) \widehat{B_{R(y)}}(\xi) \exp (2 \pi i(x-y) \xi) d \xi\right| d \mu(x) d \mu(y) .
\end{aligned}
$$

Since $\widehat{B_{R}}(\xi)$ is a radial function, an integration in polar coordinates with the abuse of notation $\widehat{B_{R(x)}}(\xi)=\widehat{B_{R(x)}}(|\xi|)$ gives

$$
\begin{aligned}
& \int_{\mathbb{R}^{d}}\left(1+|\xi|^{2}\right)^{-\gamma} \widehat{B_{R(x)}}(\xi) \widehat{B_{R(y)}}(\xi) \exp (2 \pi i(x-y) \xi) d \xi \\
& =\int_{0}^{+\infty}\left(1+\rho^{2}\right)^{-\gamma} \widehat{B_{R(x)}}(\rho) \widehat{B_{R(y)}}(\rho)\left(\int_{\{|\vartheta|=1\}} \exp (2 \pi i(x-y) \rho \vartheta) d \vartheta\right) \rho^{d-1} d \rho \\
& =(2 \pi)^{d / 2} \int_{0}^{+\infty}\left(1+\rho^{2}\right)^{-\gamma} \widehat{B_{R(x)}}(\rho) \widehat{B_{R(y)}}(\rho) \frac{J_{(d-2) / 2}(2 \pi|x-y| \rho)}{(2 \pi|x-y| \rho)^{(d-2) / 2}} \rho^{d-1} d \rho .
\end{aligned}
$$


The Bessel functions satisfy the estimates $\left|J_{\nu}(z)\right| \leq c \min \left\{|z|^{\nu},|z|^{-1 / 2}\right\}$ and, by the previous lemma, $\left|\widehat{B_{R}}(\rho)\right| \leq c R^{-\alpha-\beta}(1+|R-\rho|)^{-k}$ for every $k$. Hence,

$$
\begin{aligned}
& I(x, y)=\left|(2 \pi)^{d / 2} \int_{0}^{+\infty}\left(1+\rho^{2}\right)^{-\gamma} \widehat{B_{R(x)}}(\rho) \widehat{B_{R(y)}}(\rho) \frac{J_{(d-2) / 2}(2 \pi|x-y| \rho)}{(2 \pi|x-y| \rho)^{(d-2) / 2}} \rho^{d-1} d \rho\right| \\
& \leq c R(x)^{-\alpha-\beta} R(y)^{-\alpha-\beta} \int_{0}^{+\infty} \frac{\min \left\{1,(|x-y| \rho)^{-(d-1) / 2}\right\}}{(1+|R(x)-\rho|)^{k}(1+|R(y)-\rho|)^{k}}\left(1+\rho^{2}\right)^{-\gamma} \rho^{d-1} d \rho .
\end{aligned}
$$

If $k$ is large and if $R(x)$ and $R(y)$ are close to each other, $R(x) \leq R(y) \leq 3 R(x)$, then

$$
I(x, y) \leq c R(x)^{d-2(\alpha+\beta+\gamma)-1} \min \left\{1,(|x-y| R(x))^{-(d-1) / 2}\right\} .
$$

If $R(x)$ and $R(y)$ are far from each other, $3 R(x)<R(y)$, then one can split the domain of integration into $0<\rho \leq R(y) / 2$ and $R(y) / 2 \leq \rho<+\infty$. In the first interval, $|R(y)-\rho|$ is essentially $R(y)$, while in the second interval, $|R(x)-\rho|$ is essentially larger than $R(y)$. Thus one obtains

$$
\begin{gathered}
I(x, y) \leq c R(x)^{d-1-2(\alpha+\beta+\gamma)} \min \left\{1,(|x-y| R(x))^{-(d-1) / 2}\right\} \\
+c R(y)^{d-1-2(\alpha+\beta+\gamma)} \min \left\{1,(|x-y| R(y))^{-(d-1) / 2}\right\} .
\end{gathered}
$$

In both cases, since $(d-1) / 4 \leq \alpha+\beta+\gamma \leq(d-1) / 2$,

$$
I(x, y) \leq c R^{d-1-2(\alpha+\beta+\gamma)} \min \left\{1,(R|x-y|)^{-(d-1) / 2}\right\} \leq c|x-y|^{2(\alpha+\beta+\gamma)+1-d} .
$$

Similarly, if $\alpha+\beta+\gamma \geq(d-1) / 2$, then $|I(x, y)| \leq c$.

Part (1) of Theorem 1 follows from Lemma 4 and Lemma 6.

The idea behind the proof of (2) is quite simple. Decompose $B_{R} * f(x)$ as $B_{R} * G^{\gamma} * G^{-\gamma} * f(x)$, with $G^{\gamma}(x)$ the Bessel potentials defined by $\widehat{G^{\gamma}}(\xi)=$ $\left(1+|\xi|^{2}\right)^{-\gamma / 2}$. By Lemma [5. $\sup _{R>0}\left\{\left|\widehat{B_{R} * G} \gamma(\xi)\right|\right\} \leq c|\xi|^{-\alpha-\beta-\gamma}$ and, since $|\xi|^{-\alpha-\beta-\gamma}$ is the Fourier transform of $c|x|^{\alpha+\beta+\gamma-d}$, one may guess that the maximal function $\sup _{R>0}\left\{\left|B_{R} * f(x)\right|\right\}$ is dominated by the fractional integral $\left|G^{-\gamma} * f\right| *$ $|x|^{\alpha+\beta+\gamma-d}$. Finally,

$$
\begin{gathered}
\int_{\mathbb{R}^{d}}\left(\int_{\mathbb{R}^{d}}\left|G^{-\gamma} * f(z)\right||x-z|^{\alpha+\beta+\gamma-d} d z\right) d \mu(x) \\
\leq\left\{\int_{\mathbb{R}^{d}}\left|G^{-\gamma} * f(z)\right|^{2} d z\right\}^{1 / 2}\left\{\int_{\mathbb{R}^{d}}\left|\int_{\mathbb{R}^{d}}\right| x-\left.\left.z\right|^{\alpha+\beta+\gamma-d} d \mu(x)\right|^{2} d z\right\}^{1 / 2} \\
=\left\{\int_{\mathbb{R}^{d}}\left|G^{-\gamma} * f(z)\right|^{2} d z\right\}^{1 / 2} \\
\times\left\{\int_{\mathbb{R}^{d}} \int_{\mathbb{R}^{d}}\left(\int_{\mathbb{R}^{d}}|x-z|^{\alpha+\beta+\gamma-d}|y-z|^{\alpha+\beta+\gamma-d} d z\right) d \mu(x) d \mu(y)\right\}^{1 / 2} \\
=c\left\{\int_{\mathbb{R}^{d}}\left(1+|\xi|^{2}\right)^{\gamma}|\widehat{f}(\xi)|^{2} d x\right\}^{1 / 2}\left\{\int_{\mathbb{R}^{d}} \int_{\mathbb{R}^{d}} \frac{d \mu(x) d \mu(y)}{|x-y|^{d-2(\alpha+\beta+\gamma)}}\right\}^{1 / 2} .
\end{gathered}
$$


The details of the proof are more involved and, indeed, we shall lose an $\varepsilon$ of the exponent. In particular, we shall prove that for every $\varepsilon>0$ there exists a square integrable function $F(x)$ such that

$$
\begin{gathered}
\sup _{R>0}\left\{\left|B_{R} * f(x)\right|\right\} \leq F *|x|^{\alpha+\beta+\gamma-\varepsilon-d}, \\
\left\{\int_{\mathbb{R}^{d}}|F(z)|^{2} d z\right\}^{1 / 2} \leq c\left\{\int_{\mathbb{R}^{d}}\left|G^{-\gamma} * f(z)\right|^{2} d z\right\}^{1 / 2} .
\end{gathered}
$$

In what follows a few properties of Bessel kernels will be needed. If $\gamma \leq 0$, then $G^{\gamma}(x)$ is a tempered distribution, while if $\gamma>0$, then it is a positive integrable function. Indeed, $G^{\gamma}(x)$ can be subordinated to the heat kernel,

$$
\begin{gathered}
G^{\gamma}(x)=\int_{\mathbb{R}^{d}}\left(1+|\xi|^{2}\right)^{-\gamma / 2} \exp (2 \pi i \xi x) d x \\
=\Gamma(\gamma / 2)^{-1} \int_{0}^{+\infty}(4 \pi t)^{-d / 2} \exp \left(-|x|^{2} / 4 t\right) \exp (-t) t^{\gamma / 2-1} d t .
\end{gathered}
$$

From this representation it follows that when $0<\gamma<d$, then $G^{\gamma}(x)$ blows up as $|x|^{\gamma-d}$ at the origin and decays exponentially at infinity. When $\gamma=d$, then $G^{d}(x)$ has a logarithmic singularity - $\log (|x|)$ at the origin, and when $\gamma>d$, then $G^{\gamma}(x)$ is bounded. See [22, Chapter V].

Lemma 7. If $0<\varepsilon<\alpha+\beta+\gamma$, then there exists $c>0$ such that

$$
\sup _{R>1}\left\{\left|B_{R} * f(x)\right|\right\} \leq c G^{\alpha+\beta+\gamma-\varepsilon} *\left(\sup _{R>0}\left\{\left|S_{R}^{0} * G^{\varepsilon-\gamma} * f\right|\right\}\right)(x) .
$$

Proof. Define

$$
\begin{gathered}
P(|\xi|)=\left(1+|\xi|^{2}\right)^{(\alpha+\beta) / 2} \widehat{B_{R}}(\xi) \\
Q(t)=S_{t}^{0} * G^{\alpha+\beta} * f(x)=\int_{\{|\xi| \leq t\}}\left(1+|\xi|^{2}\right)^{-(\alpha+\beta) / 2} \widehat{f}(\xi) \exp (2 \pi i \xi x) d \xi .
\end{gathered}
$$

Observe that $P(t)$ is also a function of $R$, and $Q(t)$ is also a function of $x$. Then

$$
\begin{gathered}
B_{R} * f(x)=\int_{0}^{+\infty} P(t) d Q(t)=-\int_{0}^{+\infty} Q(t) d P(t), \\
\sup _{R>1}\left\{\left|B_{R} * f(x)\right|\right\} \leq \sup _{t>0}\{|Q(t)|\} \sup _{R>1}\left\{\int_{0}^{+\infty}\left|\frac{d}{d t} P(t)\right| d t\right\} .
\end{gathered}
$$

As in Lemma 5, it can be proved that the integral of $d P(t) / d t$ can be bounded independently on $R$. Indeed, with a small abuse of notation,

$$
\begin{aligned}
& \frac{d}{d t} P(t)=\frac{d}{d t}\left(R^{-\beta}\left(1+t^{2}\right)^{(\alpha+\beta) / 2}\left(\left(1-t^{2} / R^{2}\right)_{+}^{\alpha}-\widehat{\psi} *\left(1-t^{2} / R^{2}\right)_{+}^{\alpha}\right)\right) \\
& =(\alpha+\beta) R^{-\beta} t\left(1+t^{2}\right)^{(\alpha+\beta) / 2-1}\left(\left(1-t^{2} / R^{2}\right)_{+}^{\alpha}-\widehat{\psi} *\left(1-t^{2} / R^{2}\right)_{+}^{\alpha}\right) \\
& \quad-2 \alpha R^{-\beta-2} t\left(1+t^{2}\right)^{(\alpha+\beta) / 2}\left(\left(1-t^{2} / R^{2}\right)_{+}^{\alpha-1}-\widehat{\psi} *\left(1-t^{2} / R^{2}\right)_{+}^{\alpha-1}\right) \\
& +2 \alpha R^{-\beta-2}\left(1+|\xi|^{2}\right)^{(\alpha+\beta) / 2}|\xi|^{-1} \int_{\mathbb{R}^{d}} \xi \cdot \zeta \widehat{\psi}(\zeta)\left(1-|\xi-\zeta|^{2} / R^{2}\right)_{+}^{\alpha-1} d \zeta
\end{aligned}
$$


Observe that, if $\alpha>0$, then $\left(1-t^{2} / R^{2}\right)_{+}^{\alpha-1}$ is integrable. Then, by an analog of Lemma 5 .

$$
\begin{gathered}
(\alpha+\beta) R^{-\beta} \int_{0}^{+\infty} t\left(1+t^{2}\right)^{(\alpha+\beta) / 2-1}\left|\left(1-t^{2} / R^{2}\right)_{+}^{\alpha}-\widehat{\psi} *\left(1-t^{2} / R^{2}\right)_{+}^{\alpha}\right| d t \leq c R^{-1}, \\
2 \alpha R^{-\beta-2} \int_{0}^{+\infty} t\left(1+t^{2}\right)^{(\alpha+\beta) / 2}\left|\left(1-t^{2} / R^{2}\right)_{+}^{\alpha-1}-\widehat{\psi} *\left(1-t^{2} / R^{2}\right)_{+}^{\alpha-1}\right| d t \leq c .
\end{gathered}
$$

Similarly, also the integral of the third term is bounded by $c R^{-1}$. Finally, since $G^{\alpha+\beta}(x)=G^{\alpha+\beta+\gamma-\varepsilon} * G^{\varepsilon-\gamma}(x)$ and since $G^{\alpha+\beta+\gamma-\varepsilon}(x)$ is positive when $\alpha+\beta+$ $\gamma-\varepsilon>0$

$$
\begin{gathered}
\sup _{t>0}\{|Q(t)|\}=\sup _{R>0}\left\{\left|S_{R}^{0} * G^{\alpha+\beta} * f\right|(x)\right\} \\
\leq G^{\alpha+\beta+\gamma-\varepsilon} *\left(\sup _{R>0}\left\{\left|S_{R}^{0} * G^{\varepsilon-\gamma} * f\right|\right\}\right)(x) .
\end{gathered}
$$

Lemma 8. If $\varepsilon>0$, then there exists $c>0$ such that for every $f(x)$,

$$
\int_{\mathbb{R}^{d}} \sup _{R>0}\left\{\left|S_{R}^{0} * G^{\varepsilon-\gamma} * f(x)\right|\right\}^{2} d x \leq c \int_{\mathbb{R}^{d}}\left(1+|\xi|^{2}\right)^{\gamma}|\widehat{f}(\xi)|^{2} d \xi .
$$

Proof. One has to prove that, if $g(x)=G^{\varepsilon-\gamma} * f(x)$, then

$$
\int_{\mathbb{R}^{d}} \sup _{R>0}\left\{\left|S_{R}^{0} * g(x)\right|\right\}^{2} d x \leq c \int_{\mathbb{R}^{d}}\left(1+|\xi|^{2}\right)^{\varepsilon}|\widehat{g}(\xi)|^{2} d \xi .
$$

Indeed, it has been proved in [6] that more is true:

$$
\int_{\mathbb{R}^{d}} \sup _{R>0}\left\{\left|S_{R}^{0} * g(x)\right|\right\}^{2} d x \leq c \int_{\mathbb{R}^{d}}|\widehat{g}(\xi)|^{2} \log ^{2}\left(e+|\xi|^{2}\right) d \xi .
$$

See also [11] and [13] for a simple proof based on the Rademacher-Menshov theorem.

Lemma 9. For every square integrable function $F(x)$, for every nonnegative finite Borel measure $d \mu(x)$, and for every $\eta>0$,

$$
\int_{\mathbb{R}^{d}}\left|G^{\eta} * F(x)\right| d \mu(x) \leq\left\{\int_{\mathbb{R}^{d}}|F(x)|^{2} d x\right\}^{1 / 2}\left\{\int_{\mathbb{R}^{d}} \int_{\mathbb{R}^{d}} G^{2 \eta}(x-y) d \mu(x) d \mu(y)\right\}^{1 / 2} .
$$

Proof. If $g(x)=\overline{G^{\eta} * F(x)} /\left|G^{\eta} * F(x)\right|$, then

$$
\begin{gathered}
\int_{\mathbb{R}^{d}}\left|G^{\eta} * F(x)\right| d \mu(x)=\int_{\mathbb{R}^{d}} \int_{\mathbb{R}^{d}} g(x)\left(1+|\xi|^{2}\right)^{-\eta / 2} \widehat{F}(\xi) \exp (2 \pi i \xi x) d \xi d \mu(x) \\
\leq\left\{\int_{\mathbb{R}^{d}}|\widehat{F}(\xi)|^{2} d \xi\right\}^{1 / 2}\left\{\int_{\mathbb{R}^{d}}\left|\int_{\mathbb{R}^{d}} g(x)\left(1+|\xi|^{2}\right)^{-\eta / 2} \exp (2 \pi i \xi x) d \mu(x)\right|^{2} d \xi\right\}^{1 / 2} \\
\leq\left\{\int_{\mathbb{R}^{d}}|F(x)|^{2} d x\right\}^{1 / 2} \\
\times\left\{\int_{\mathbb{R}^{d}} \int_{\mathbb{R}^{d}}\left|\int_{\mathbb{R}^{d}}\left(1+|\xi|^{2}\right)^{-\eta} \exp (2 \pi i(x-y) \xi) d \xi\right| d \mu(x) d \mu(y)\right\}^{1 / 2} \\
=\left\{\int_{\mathbb{R}^{d}}|\widehat{F}(\xi)|^{2} d \xi\right\}^{1 / 2}\left\{\int_{\mathbb{R}^{d}} \int_{\mathbb{R}^{d}} G^{2 \eta}(x-y) d \mu(x) d \mu(y)\right\}^{1 / 2}
\end{gathered}
$$


The proof of (2) in Theorem 1 follows from Lemma 7 Lemma 8 , and Lemma 9 with $F(x)=\sup _{R>0}\left\{\left|S_{R}^{0} * G^{\varepsilon-\gamma} * f\right|\right\}, \eta=\alpha+\beta+\gamma-\varepsilon, 0<\varepsilon<\alpha+\beta+\gamma$. It suffices to recall that, if $0<2 \eta<d$, then the Bessel kernel $G^{2 \eta}(x)$ blows up as $|x|^{2 \eta-d}$ at the origin and decays exponentially at infinity.

Finally, (3) is a simple generalization of the classical Bochner result, which follows from the estimate

$$
\begin{gathered}
\left|B_{R} * f(x)\right|=\left|B_{R} * G^{\gamma} * G^{-\gamma} * f(x)\right| \\
\leq\left\{\int_{\mathbb{R}^{d}}\left|B_{R} * G^{\gamma}(x)\right|^{2} d x\right\}^{1 / 2}\left\{\int_{\mathbb{R}^{d}}\left|G^{-\gamma} * f(x)\right|^{2} d x\right\}^{1 / 2} \\
=\left\{\int_{\mathbb{R}^{d}}\left(1+|\xi|^{2}\right)^{-\gamma}\left|\widehat{B_{R}}(\xi)\right|^{2} d \xi\right\}^{1 / 2}\left\{\int_{\mathbb{R}^{d}}\left(1+|\xi|^{2}\right)^{\gamma}|\widehat{f}(\xi)|^{2} d \xi\right\}^{1 / 2} .
\end{gathered}
$$

Indeed, it follows from Lemma 5 that, if $\alpha+\beta+\gamma \geq(d-1) / 2$, then

$$
\sup _{R>0}\left\{\int_{\mathbb{R}^{d}}\left(1+|\xi|^{2}\right)^{-\gamma}\left|\widehat{B_{R}}(\xi)\right|^{2} d \xi\right\}^{1 / 2}<+\infty .
$$

Proof of Corollary 2. In order to prove (1) or (2), it suffices to show that the maximal function $\sup _{R>0}\left\{R^{-\beta}\left|S_{R}^{\alpha} * f(x)\right|\right\}$ cannot be infinite on subsets of $\Omega$ with Hausdorff dimension larger than $\delta$. A set of dimension $\eta$ has infinite $\sigma$-dimensional measure for every $\sigma<\eta$ and, by Frostman's lemma, it supports a positive measure with $\mu\{|x-p|<r\} \leq r^{\sigma}$, and this measure has finite $\tau$ energy for every $\tau<\sigma$. See [16, Chapter 8]. It then suffices to apply part (1) or part (2) of Theorem 1 with $\delta<\tau<\sigma<\eta$. Similarly, (3) follows from part (3) of the theorem.

The proof of Theorem 3 is inspired by [15, which shows that for every set of measure zero there is a continuous function with Fourier series diverging on it. Some auxiliary results are needed. The first lemma states that a countable union of sets of nonlocalization is a set of nonlocalization.

Lemma 10. Let $\Omega$ be an open set, $A$ a bounded open subset of $\Omega$, and $\left\{B_{n}\right\}_{n=1}^{+\infty}$ a sequence of compact subsets of $A$, with distance $(A, \partial \Omega)>\varepsilon$ and distance $\left(B_{n}, \partial A\right)>\varepsilon$, for some $\varepsilon>0$. Assume that for every $n$ there exists a square integrable function $h_{n}(x)$ with support off $\Omega$ and with

$$
\sup _{R>0}\left\{R^{-\beta}\left|S_{R}^{\alpha} * h_{n}(x)\right|\right\}=+\infty \text { for every } x \text { in } B_{n} .
$$

Then there exists a square integrable function $f(x)$ with support off $A$ and with

$$
\sup _{R>0}\left\{R^{-\beta}\left|S_{R}^{\alpha} * f(x)\right|\right\}=+\infty \text { for every } x \text { in } \bigcup_{n=1}^{+\infty} B_{n} .
$$

Proof. Assume that in the sequence $\left\{B_{n}\right\}_{n=1}^{+\infty}$ each compact set is repeated infinitely many times,

$$
\left\{B_{1}, B_{2}, B_{1}, B_{2}, B_{3}, B_{1}, B_{2}, B_{3}, B_{4}, B_{1}, B_{2}, B_{3}, B_{4}, B_{5}, \ldots\right\} .
$$

Also assume that $\left\{\int_{\mathbb{R}^{d}}\left|h_{n}(x)\right|^{2} d x\right\}^{1 / 2} \leq 1$. Let $\chi(\xi)$ be a radial nonnegative smooth function, with $0 \leq \chi(\xi) \leq 1, \chi(\xi)=1$ if $|\xi| \leq 1 / 3$ and $\chi(\xi)=0$ if $|\xi| \geq 2 / 3$. 
Decompose $S_{R}^{\alpha}(x)$ into $U_{R}(x)+V_{R}(x)$, with

$$
\begin{gathered}
\widehat{U_{R}}(\xi)=\chi\left(R^{-1} \xi\right)\left(1-|\xi|^{2} / R^{2}\right)_{+}^{\alpha}, \\
\widehat{V_{R}}(\xi)=\left(1-\chi\left(R^{-1} \xi\right)\right)\left(1-|\xi|^{2} / R^{2}\right)_{+}^{\alpha} .
\end{gathered}
$$

Since $\widehat{U_{R}}(\xi)$ is smooth, $U_{R}(x)$ has fast decay at infinity, and for every $x$ in $\Omega$,

$$
\limsup _{R \rightarrow+\infty}\left\{R^{-\beta}\left|U_{R} * h_{n}(x)\right|\right\}=0 .
$$

Hence,

$$
\sup _{R>0}\left\{R^{-\beta}\left|V_{R} * h_{n}(x)\right|\right\}=+\infty \text { for every } x \text { in } B_{n} .
$$

Set $R_{0}=1$ and, by induction, define $\left\{\tau_{n}\right\}_{n=1}^{+\infty},\left\{R_{n}\right\}_{n=1}^{+\infty}$, by

$$
\begin{gathered}
\tau_{n}>3^{n}, \quad R_{n}>12 R_{n-1}, \\
\sup _{12 R_{n-1} \leq R \leq R_{n}}\left\{R^{-\beta}\left|V_{R} * h_{n}(x)\right|\right\}>\tau_{n} \text { for every } x \text { in } B_{n} .
\end{gathered}
$$

Also define $\left\{k_{n}(x)\right\}_{n=1}^{+\infty}$ by

$$
\widehat{k_{n}}(\xi)=\left(\chi\left(\left(3 R_{n}\right)^{-1} \xi\right)-\chi\left(\left(6 R_{n-1}\right)^{-1} \xi\right)\right) \widehat{h_{n}}(\xi) .
$$

Observe that

$$
\left\{\int_{\mathbb{R}^{d}}\left|k_{n}(x)\right|^{2} d x\right\}^{1 / 2} \leq\left\{\int_{\mathbb{R}^{d}}\left|h_{n}(x)\right|^{2} d x\right\}^{1 / 2} .
$$

Also observe that

$$
\chi\left(\left(3 R_{n}\right)^{-1} \xi\right)-\chi\left(\left(6 R_{n-1}\right)^{-1} \xi\right)= \begin{cases}0 & \text { if }|\xi| \leq 2 R_{n-1} \text { or }|\xi| \geq 2 R_{n} \\ 1 & \text { if } 4 R_{n-1} \leq|\xi| \leq R_{n}\end{cases}
$$

Hence, if $12 R_{n-1} \leq R \leq R_{n}$, then

$$
V_{R} * k_{m}(x)=\left\{\begin{array}{l}
V_{R} * h_{n}(x) \text { if } m=n, \\
0 \text { if } m \neq n .
\end{array}\right.
$$

Fix a smooth function $\psi(x)$ with support in $\Omega$, with $\psi(x)=1$ if $x$ is in $A$ and $\psi(x)=0$ if distance $\{x, A\} \geq \varepsilon / 2$. Define

$$
f(x)=\sum_{n=1}^{+\infty} 2^{-n}(1-\psi(x)) k_{n}(x), \quad g(x)=\sum_{n=1}^{+\infty} 2^{-n} \psi(x) k_{n}(x) .
$$

In order to prove that $R^{-\beta}\left|V_{R} * f(x)\right|$ is unbounded on $\bigcup_{n=1}^{+\infty} B_{n}$, it suffices to show that $R^{-\beta}\left|V_{R} *(f+g)(x)\right|$ is unbounded on $\bigcup_{n=1}^{+\infty} B_{n}$, while $R^{-\beta}\left|V_{R} * g(x)\right|$ is bounded everywhere. If $12 R_{n-1} \leq R \leq R_{n}$, then

$$
V_{R} *(f+g)(x)=V_{R} *\left(\sum_{m=1}^{+\infty} 2^{-m} k_{m}\right)(x)=2^{-n} V_{R} * h_{n}(x) .
$$

Hence,

$$
\sup _{12 R_{n-1} \leq R \leq R_{n}}\left\{R^{-\beta}\left|V_{R} *(f+g)(x)\right|\right\}>2^{-n} \tau_{n} \quad \text { for every } x \text { in } B_{n} .
$$


Finally, since in $\left\{B_{n}\right\}_{n=1}^{+\infty}$ each compact set is repeated infinitely many times,

$$
\sup _{R>0}\left\{R^{-\beta}\left|V_{R} *(f+g)(x)\right|\right\}=+\infty \text { for every } x \text { in } \bigcup_{n=1}^{+\infty} B_{n} .
$$

Now estimate $V_{R} * g(x)$. If $H_{R}(x)=R^{d} H(R x)$ is the kernel associated to the multiplier $\chi\left(R^{-1} \xi\right)$, then

$$
\begin{gathered}
V_{R} * g(x)=\sum_{n=1}^{+\infty} 2^{-n} \int_{\mathbb{R}^{d}}\left(\int_{\mathbb{R}^{d}} V_{R}(x-y) H_{3 R_{n}}(y-z) \psi(y) d y\right) h_{n}(z) d z \\
-\sum_{n=1}^{+\infty} 2^{-n} \int_{\mathbb{R}^{d}}\left(\int_{\mathbb{R}^{d}} V_{R}(x-y) H_{6 R_{n-1}}(y-z) \psi(y) d y\right) h_{n}(z) d z .
\end{gathered}
$$

The two series are similar and it suffices to consider the first one. In order to estimate the inner integral, fix an integer $j$ and define a new kernel $W_{R}(x)=$ $R^{d} W(R x)$ by

$$
\widehat{W_{R}}(\xi)=\left(4 \pi^{2}\left|R^{-1} \xi\right|^{2}\right)^{-j}\left(1-\chi\left(R^{-1} \xi\right)\right)\left(1-|\xi|^{2} / R^{2}\right)_{+}^{\alpha} .
$$

If $\Delta=-\sum_{i=1}^{d} \partial^{2} / \partial x_{i}^{2}$ is the Laplace operator, then $V_{R}(x)=R^{-2 j} \Delta^{j} W_{R}(x)$ and an integration by parts gives

$$
\begin{gathered}
\int_{\mathbb{R}^{d}} V_{R}(x-y) H_{3 R_{n}}(y-z) \psi(y) d y \\
=R^{-2 j} \int_{\mathbb{R}^{d}} \Delta^{j} W_{R}(x-y) H_{3 R_{n}}(y-z) \psi(y) d y \\
=R^{d-2 j}\left(3 R_{n}\right)^{d} \int_{\mathbb{R}^{d}} W(R(x-y)) \Delta^{j}\left(H\left(3 R_{n}(y-z)\right) \psi(y)\right) d y .
\end{gathered}
$$

First of all, $|W(R(x-y))| \leq c$. Moreover, since the function $\psi(y)$ is smooth with compact support and since all derivatives of the kernel $H_{R}(x)$ have fast decay at infinity, for every $r>0$ one has

$$
\left|\Delta^{j}\left(H\left(3 R_{n}(y-z)\right) \psi(y)\right)\right| \leq c R_{n}^{2 j-r}|y-z|^{-r} .
$$

Since $\psi(y)$ has support in $\{$ distance $\{y, A\} \leq \varepsilon / 2\}$, this implies that

$$
\begin{gathered}
\quad\left|\int_{\mathbb{R}^{d}} V_{R}(x-y) H_{3 R_{n}}(y-z) \psi(y) d y\right| \\
\leq c R^{d-2 j} R_{n}^{d+2 j-r} \int_{\{\text {distance }\{y, A\} \leq \varepsilon / 2\}}|y-z|^{-r} d y .
\end{gathered}
$$

Hence, if $j \geq(-\beta+d) / 2$ and $r \geq d+2 j$,

$$
\begin{gathered}
\leq c \sum_{n=1}^{+\infty} 2^{-n} \int_{\mathbb{R}^{d}}\left(\int_{\{\text {distance }\{y, A\} \leq \varepsilon / 2\}}|y-z|^{-r} d y\right)\left|h_{n}(z)\right| d z \\
\leq c \sum_{n=1}^{+\infty} 2^{-n}\left\{\int_{\mathbb{R}^{d}-\Omega}\left|\int_{\{\text {distance }\{y, A\} \leq \varepsilon / 2\}}\right| y-\left.\left.z\right|^{-r} d y\right|^{2} d z\right\}^{1 / 2}\left\{\int_{\mathbb{R}^{d}}\left|h_{n}(z)\right|^{2} d z\right\}^{1 / 2} \\
\leq c .
\end{gathered}
$$


The second result needed in the proof of Theorem 3 is a construction of bump functions supported in long and thin tubes, with Riesz means suitably large in disjoint adjacent tubes. The following lemma is similar to Lemma 1 in 8 ].

Lemma 11. Let $\varphi(x)$ be a smooth bump function supported in $\{|x|<1\}$, and let $\varphi_{R}(x)=\exp \left(2 \pi i R x_{1}\right) \varphi\left(x_{1}-3, R^{1 / 2} x^{\prime}\right)$, where $R>1$ and $x=\left(x_{1}, x^{\prime}\right) \in$ $\mathbb{R} \times \mathbb{R}^{d-1}$. Then the following hold:

(1) The function $\varphi_{R}(x)$ has support in the tube $\left\{\left|x_{1}-3\right| \leq 1,\left|x^{\prime}\right| \leq R^{-1 / 2}\right\}$.

(2) There exists a constant $C>0$ such that in the tube $\left\{\left|x_{1}\right| \leq 1,\left|x^{\prime}\right| \leq R^{-1 / 2}\right\}$,

$$
\left|S_{R}^{\alpha} * \varphi_{R}(x)\right| \geq C R^{-\alpha} .
$$

(3) For every $k>0$ there is a constant $c$ such that for all $x$,

$$
\left|S_{R}^{\alpha} * \varphi_{R}(x)\right| \leq c R^{-\alpha}\left(1+R^{1 / 2}\left|x^{\prime}\right|\right)^{-k} .
$$

(4) For every $k>0$ there is a constant $c$ such that for all $|x|<1$ and $T / R \notin$ $(1 / 2,2)$,

$$
\left|S_{T}^{\alpha} * \varphi_{R}(x)\right| \leq c(\max \{R, T\})^{-k} .
$$

Proof. The kernel $S_{T}^{\alpha}(x)$ oscillates as $T^{(d-1) / 2-\alpha}|x|^{-\alpha-(d+1) / 2} \exp (2 \pi i T|x|)$, and when $|x|<1$, then $S_{T}^{\alpha} * \varphi_{R}(x)$ is essentially

$$
\begin{aligned}
R^{-(d-1) / 2} T^{(d-1) / 2-\alpha} & \int_{\mathbb{R}^{d}} \varphi\left(y_{1}-3, y^{\prime}\right) \\
& \exp \left(2 \pi i\left(R y_{1}+T \sqrt{\left(x_{1}-y_{1}\right)^{2}+\left|x^{\prime}-R^{-1 / 2} y^{\prime}\right|^{2}}\right)\right) d y .
\end{aligned}
$$

The phase $y \rightsquigarrow R y_{1}+T \sqrt{\left(x_{1}-y_{1}\right)^{2}+\left|x^{\prime}-R^{-1 / 2} y^{\prime}\right|^{2}}$ has critical points in the support of $\varphi\left(y_{1}-3, y^{\prime}\right)$ only if $R=T$ and $\left|x^{\prime}\right| \leq R^{-1 / 2}$, and the lemma follows from standard estimates for oscillating integrals.

Proof of Theorem 3. With the notation of the above lemma, $\varphi_{R}(x)$ is square integrable with norm about $R^{-(d-1) / 4}$ and support in $\left\{\left|x_{1}-3\right| \leq 1,\left|x^{\prime}\right| \leq R^{-1 / 2}\right\}$, and $\left|S_{R}^{\alpha} * \varphi_{R}(x)\right| \geq c R^{-\alpha}$ in $\left\{\left|x_{1}-3\right| \leq 1,\left|x^{\prime}\right| \leq R^{-1 / 2}\right\}$. Hence, if $f(x)=$ $\sum_{n=1}^{+\infty} n^{-2} 2^{n(d-1) / 4} \varphi_{2^{n}}(x)$, then $f(x)$ is square integrable with support in $\left\{\left|x_{1}-3\right|\right.$ $\left.\leq 1,\left|x^{\prime}\right| \leq 1\right\}$. Moreover, if $\left|x_{1}-3\right| \leq 1, x^{\prime}=0, R=2^{m}$, then

$$
\begin{gathered}
R^{-\beta}\left|S_{R}^{\alpha} * f(x)\right| \\
\geq m^{-2} 2^{m((d-1) / 4-\beta)}\left|S_{2^{m}}^{\alpha} * \varphi_{2^{m}}(x)\right|-2^{-\beta m} \sum_{n \neq m} n^{-2} 2^{n(d-1) / 4}\left|S_{2^{m}}^{\alpha} * \varphi_{2^{n}}(x)\right| \\
\geq c m^{-2} 2^{m((d-1) / 4-\alpha-\beta)}-c 2^{-\beta m} \sum_{n \neq m} n^{-2} 2^{n(d-1) / 4}\left(\max \left\{2^{m}, 2^{n}\right\}\right)^{-k} .
\end{gathered}
$$

Hence, if $\alpha+\beta<(d-1) / 4$, then $\sup _{R>0}\left\{R^{-\beta}\left|S_{R}^{\alpha} * f(x)\right|\right\}=+\infty$ on the segment $\left\{\left|x_{1}\right| \leq 1, \quad\left|x^{\prime}\right|=0\right\}$. This shows that when $\alpha+\beta<(d-1) / 4$, the sets of nonlocalization can have dimension 1 .

The construction of sets of nonlocalization with dimension $\delta>1$ is similar, only more complicated. Let $\left\{\beta_{n}\right\}$ be a positive sequence tending very slowly to infinity and let $\left\{\theta_{n}\right\}$ be a positive sequence such that $\sum_{n=1}^{+\infty} \beta_{n} \theta_{n}^{1 / 2}$ converges. By hypothesis, one can find a countable family of tubes $\mathcal{F}=\left\{T_{j}\right\}$, with axis parallel 
to the first coordinate axis and of length 1 , and with $\sum_{j=1}^{+\infty} w\left(T_{j}\right)^{\delta-1} \leq \varepsilon \theta_{1}$, where $w\left(T_{j}\right)$ denotes the cross section diameter of the tube $T_{j}$ and $\varepsilon$ is a small dimensional constant. One can also assume that $\mathcal{F}$ covers $E$ infinitely often, and also that the sequence $\left\{w\left(T_{j}\right)\right\}$ is nonincreasing. We want to decompose the set $E$ into a finite union of sets $\left\{E^{l}\right\}$ with a sort of Cantor structure. For a large constant, $M$, altering $\varepsilon$ by a dimensional constant, one may assume that each $T_{j}$ has size $1 \times M^{-k} \times M^{-k} \times \cdots \times M^{-k}$, for some integer $k$ which depends on the tube. Moreover, one may assume that if two tubes have the same cross section $M^{-k}$, then they are disjoint. Let $D$ be a large constant. We partition $\mathcal{F}$ into subfamilies $\left\{\mathcal{F}_{l}\right\}_{l=1}^{D^{d-1}}$ with the property that parallel congruent tubes in any $\mathcal{F}_{l}$ of any given size are separated by a factor $D$ times their cross section. Let $E^{l}$ be the set of those $x \in E$ which are in infinitely many members of $\mathcal{F}_{l}$. Since every $x \in E$ is in infinitely many members of $\mathcal{F}$, each $x \in E$ belongs to some $E^{l}$. Since finite unions of sets of divergence are sets of divergence, it suffices to show that each $E^{l}$ is a set of divergence.

Rename a typical $E^{l}$ as $E$. Now let $\left\{j_{m}\right\}$ be a subsequence of $\mathbb{N}$ such that $\sum_{j=j_{m}}^{+\infty} w\left(T_{j}\right)^{\delta-1} \leq \theta_{m}$. Also let $\mathcal{A}_{m}=\left\{j_{m}, j_{m}+1, \ldots, j_{m+1}-1\right\}, \mathcal{T}_{m}=\left\{T_{j}\right.$ : $\left.j \in \mathcal{A}_{m}\right\}$, and $E_{m}=\bigcup_{j \in \mathcal{A}_{m}} T_{j}$. Since the $w\left(T_{j}\right)$ are decreasing, by replacing the sequence $\left\{j_{m}\right\}$ by one tending more rapidly to infinity, one can assume that no two congruent tubes lie in different $\mathcal{T}_{m}$ 's. Now let $T$ be any tube of width $w(T)=R^{-1 / 2}$, and denote by $\varphi^{T}(x)$ the translation of the function $\exp \left(2 \pi i R x_{1}\right) \varphi\left(x_{1}-3, R^{1 / 2} x^{\prime}\right)$ from the above lemma which is adapted to the tube $T+(3,0)$. For every $m$, there is a choice of \pm such that

$$
\begin{aligned}
\int_{\mathbb{R}^{d}} \mid \sum_{T \in \mathcal{T}_{m}} & \pm\left. w(T)^{-2(\alpha+\beta)} \varphi^{T}(x)\right|^{2} d x \leq \sum_{T \in \mathcal{T}_{m}} \int_{\mathbb{R}^{d}}\left|w(T)^{-2(\alpha+\beta)} \varphi^{T}(x)\right|^{2} d x \\
\leq C \sum_{T \in \mathcal{T}_{m}} w(T)^{d-1-4(\alpha+\beta)} & \leq C \sum_{T \in \mathcal{T}_{m}} w(T)^{\delta-1} \leq C \theta_{m} .
\end{aligned}
$$

Set $f_{m}(x)=\sum_{T \in \mathcal{T}_{m}} \pm w(T)^{-2(\alpha+\beta)} \varphi^{T}(x)$ for the choice of \pm . Then for each $x \in E_{m}$, there is a $T_{x}$ such that $x \in T_{x} \in \mathcal{T}_{m}, T_{x}$ has size $1 \times R^{-1 / 2} \times \cdots \times R^{-1 / 2}$ for some $R$ depending on $x$ and $m$ and, by the above lemma,

$$
\left|S_{R}^{\alpha} * f_{m}(x)\right| \geq C R^{-\alpha} w\left(T_{x}\right)^{-2(\alpha+\beta)}=R^{\beta} .
$$

Moreover, if $n \neq m$, then the members of $\mathcal{T}_{n}$ differ in cross-sectional diameter from those of $\mathcal{T}_{m}$ by a multiplicative factor of at least $M^{|n-m|}$ and $S_{R}^{\alpha} * f_{n}(x)$ is negligible. Set $f(x)=\sum_{n=1}^{+\infty} \beta_{n} f_{n}(x)$. Then $f(x)$ is square integrable with support disjoint from $\{|x|<1\}$. Moreover, by the previous remarks, if $x \in E_{m}$, there is an $R$ such that $R^{-\beta}\left|S_{R}^{\alpha} * f(x)\right| \sim \beta_{m} R^{-\beta}\left|S_{R}^{\alpha} * f_{m}(x)\right| \geq C \beta_{m}$, provided the sequence $\left\{\beta_{n}\right\}$ goes to infinity very slowly. Now if $x \in E$, there is a sequence $m_{k}$ such that $x \in \bigcap_{k=1}^{+\infty} E_{m_{k}}$, thus there is a sequence $R_{m_{k}}$, depending on $x$, such that $R_{m_{k}}^{-\beta}\left|S_{R_{m_{k}}}^{\alpha} * f(x)\right| \geq C \beta_{m_{k}}$ for all $k$, and so $\sup _{R>0}\left\{R^{-\beta}\left|S_{R}^{\alpha} * f(x)\right|\right\}=+\infty$.

We would like to conclude with a couple of remarks.

There is a sort of analogue of the above pointwise localization for localization in norm. If a square integrable function vanishes in an open set, then the Riesz means converge inside this set with respect to some $L(p)$ norm with $p>2$. Indeed, using the decomposition of the kernel associated to the Riesz means into a 
kernel with compact support $\psi(x) S_{R}^{\alpha}(x)$ and a kernel with small Fourier transform $(1-\psi(x)) S_{R}^{\alpha}(x)$ and some restriction properties of Fourier transforms to surfaces with positive Gauss curvature ([23. Chapter VIII $]$ ), one can prove that if a square integrable function $f(x)$ vanishes in an open set $\Omega$, then

$$
\lim _{R \rightarrow+\infty} R^{-\beta}\left\{\int_{\{x \in \Omega, \operatorname{distance}\{x, \partial \Omega\}>\varepsilon>0\}}\left|S_{R}^{\alpha} * f(x)\right|^{p} d x\right\}^{1 / p}=0,
$$

where

$$
\left\{\begin{array}{l}
\alpha+\beta=(d-1)(p-2) / 4 p \quad \text { if } 2 \leq p \leq(2 d+2) /(d-1) \\
\alpha+\beta=(d-1) / 2-d / p \quad \text { if }(2 d+2) /(d-1) \leq p \leq+\infty .
\end{array}\right.
$$

In particular, observe that when $\alpha+\beta \geq(d-1) / 2$, then one can take $p=+\infty$, which is the Bochner result.

Finally, some of the above results for trigonometric expansions on Euclidean spaces can be easily transferred to eigenfunction expansions on manifolds. See, for example, [4, 21] for a wave equation approach to this transference.

\section{REFERENCES}

[1] Juan Antonio Barceló, Jonathan Bennett, Anthony Carbery, and Keith M. Rogers, On the dimension of divergence sets of dispersive equations, Math. Ann. 349 (2011), no. 3, 599-622, DOI 10.1007/s00208-010-0529-z. MR2754999 (2012a:35260)

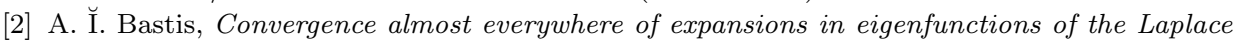
operator on a sphere (Russian), Mat. Zametki 33 (1983), no. 6, 857-862. MR709224 (84h:35123)

[3] A. I. Bastis, The generalized localization principle for an $N$-multiple Fourier integral in classes $L_{p}$ (Russian), Dokl. Akad. Nauk SSSR 304 (1989), no. 3, 526-529; English transl., Soviet Math. Dokl. 39 (1989), no. 1, 91-94. MR989167 (90g:42027)

[4] Luca Brandolini and Leonardo Colzani, Decay of Fourier transforms and summability of eigenfunction expansions, Ann. Scuola Norm. Sup. Pisa Cl. Sci. (4) 29 (2000), no. 3, 611638. MR $1817712(2002 \mathrm{e}: 35178)$

[5] Anthony Carbery, José L. Rubio de Francia, and Luis Vega, Almost everywhere summability of Fourier integrals, J. London Math. Soc. (2) 38 (1988), no. 3, 513-524. MR.972135 (90e:42033)

[6] Anthony Carbery and Fernando Soria, Almost-everywhere convergence of Fourier integrals for functions in Sobolev spaces, and an $L^{2}$-localisation principle, Rev. Mat. Iberoamericana 4 (1988), no. 2, 319-337, DOI 10.4171/RMI/76. MR1028744 (91d:42015)

[7] Anthony Carbery and Fernando Soria, Sets of divergence for the localization problem for Fourier integrals (English, with English and French summaries), C. R. Acad. Sci. Paris Sér. I Math. 325 (1997), no. 12, 1283-1286, DOI 10.1016/S0764-4442(97)82354-3. MR.1490414 (98h:42013)

[8] Anthony Carbery and Fernando Soria, Pointwise Fourier inversion and localisation in $\mathbf{R}^{n}$, Proceedings of the conference dedicated to Professor Miguel de Guzmán (El Escorial, 1996), 1997, pp. 847-858, DOI 10.1007/BF02656490. MR1600203 (99c:42018)

[9] Anthony Carbery, Fernando Soria, and Ana Vargas, Localisation and weighted inequalities for spherical Fourier means, J. Anal. Math. 103 (2007), 133-156, DOI 10.1007/s11854-0080004-x. MR2373266 (2009g:42019)

[10] Leonardo Colzani, Regularity of spherical means and localization of spherical harmonic expansions, J. Austral. Math. Soc. Ser. A 41 (1986), no. 3, 287-297. MR855392 (88c:43010)

[11] Leonardo Colzani, Time decay and regularity of solutions to the wave equation and convergence of Fourier expansions, J. Fourier Anal. Appl. 9 (2003), no. 1, 49-66, DOI 10.1007/s00041-003-0002-4. MR.1953072 (2003k:35142)

[12] Leonardo Colzani, Fourier expansions of functions with bounded variation of several variables, Trans. Amer. Math. Soc. 358 (2006), no. 12, 5501-5521 (electronic), DOI 10.1090/S0002-9947-06-03910-9. MR2238924 (2007c:42017) 
[13] Leonardo Colzani, Christopher Meaney, and Elena Prestini, Almost everywhere convergence of inverse Fourier transforms, Proc. Amer. Math. Soc. 134 (2006), no. 6, 1651-1660 (electronic), DOI 10.1090/S0002-9939-05-08329-2. MR2204276 (2006j:42015)

[14] Leonardo Colzani and Marco Vignati, The Gibbs phenomenon for multiple Fourier integrals, J. Approx. Theory 80 (1995), no. 1, 119-131, DOI 10.1006/jath.1995.1007. MR1308597 (95k:42021)

[15] Jean-Pierre Kahane and Yitzhak Katznelson, Sur les ensembles de divergence des séries trigonométriques (French), Studia Math. 26 (1966), 305-306. MR0199633 (33 \#7776)

[16] Pertti Mattila, Geometry of sets and measures in Euclidean spaces, Cambridge Studies in Advanced Mathematics, vol. 44, Cambridge University Press, Cambridge, 1995. Fractals and rectifiability. MR 1333890 (96h:28006)

[17] Christopher Meaney, Divergent Jacobi polynomial series, Proc. Amer. Math. Soc. 87 (1983), no. 3, 459-462, DOI 10.2307/2043632. MR684639 (84c:42040)

[18] Christopher Meaney, Localization of spherical harmonic expansions, Monatsh. Math. 98 (1984), no. 1, 65-74, DOI 10.1007/BF01536909. MR771835 (86f:58160)

[19] Emmanuel Montini, On the capacity of sets of divergence associated with the spherical partial integral operator, Trans. Amer. Math. Soc. 355 (2003), no. 4, 1415-1441 (electronic), DOI 10.1090/S0002-9947-02-03144-6. MR1946398(2003h:42020)

[20] Mark A. Pinsky, Fourier inversion for piecewise smooth functions in several variables, Proc. Amer. Math. Soc. 118 (1993), no. 3, 903-910, DOI 10.2307/2160140. MR1146865(93i:42017)

[21] Mark A. Pinsky and Michael E. Taylor, Pointwise Fourier inversion: a wave equation approach, J. Fourier Anal. Appl. 3 (1997), no. 6, 647-703, DOI 10.1007/BF02648262. MR 1481629(99d:42019)

[22] Elias M. Stein, Singular integrals and differentiability properties of functions, Princeton Mathematical Series, No. 30, Princeton University Press, Princeton, N.J., 1970. MR0290095 $(44$ \#7280)

[23] Elias M. Stein, Harmonic analysis: real-variable methods, orthogonality, and oscillatory integrals, Princeton Mathematical Series, vol. 43, Princeton University Press, Princeton, NJ, 1993. With the assistance of Timothy S. Murphy; Monographs in Harmonic Analysis, III. MR.1232192 (95c:42002)

[24] Elias M. Stein and Guido Weiss, Introduction to Fourier analysis on Euclidean spaces, Princeton University Press, Princeton, N.J., 1971. Princeton Mathematical Series, No. 32. MR0304972(46 \#4102)

[25] A. Zygmund, Trigonometric series. Vol. I, II, 3rd ed., Cambridge Mathematical Library, Cambridge University Press, Cambridge, 2002. With a foreword by Robert A. Fefferman. MR:1963498(2004h:01041)

Dipartimento di Matematica e Applicazioni, Università di Milano Bicocca, Via R.Cozzi 53, 20125 Milano, Italia

E-mail address: leonardo.colzani@unimib.it

Dipartimento di Ingegneria dell'Informazione e Metodi Matematici, Università di Bergamo, Viale Marconi 5, 24044 Dalmine (BG), Italia

E-mail address: giacomo.gigante@unibg.it

Departamento de Matemáticas, Universidad Autónoma de Madrid, 28049 Madrid, ESPAÑA

E-mail address: ana.vargas@uam.es 